\title{
Gender and Psychotropic Poisoning in the USA
}

\author{
Aleena Vargas ${ }^{\mathrm{a}, \mathrm{c}, \mathrm{d}}$, George Ormseth ${ }^{\mathrm{a}, \mathrm{c}}$, Ali Seifi ${ }^{\mathrm{b}}$
}

\begin{abstract}
Background: This study focuses on gender-based trends in psychotropic agent poisoning across a 17-year span. The goal of the study was to determine whether there are statistically significant differences in the characteristics of male and female hospital stays for psychotropic agent poisoning.
\end{abstract}

Methods: We used the Healthcare Cost and Utilization Project (HCUP) national database to perform a retrospective cohort study analyzing trends for poisoning by psychotropic agents in males and females between 1997 and 2014.

Results: Between 1997 and 2014, HCUP recorded a total of 1,368,649 psychotropic agent poisoning discharges. The overall number of discharges increased from 62,148 to $82,905(\mathrm{P}<0.001)$. The average age at discharge increased from 37.36 to 40.85 years $(\mathrm{P}<0.001)$. As the average length of stay increased from 2.3 to 3.2 days $(\mathrm{P}<0.001)$, hospital charges increased from $\$ 6,357$ to $\$ 27,892$ ( $\mathrm{P}<0.001)$. Across the study period, the number of in-hospital deaths increased from 468 to 755 ( $\mathrm{P}<0.001)$. In each year of the study, both number of discharges and average age were found to be greater for females than for males $(\mathrm{P}<0.001)$

Conclusions: Female discharges were consistently higher than male discharges, suggesting that female patients were more likely to experience psychotropic poisoning than male patients. This may possibly be due to a greater number of women being prescribed psychotropic medications, in concordance with higher rates of mood and anxiety disorders. Additionally, there exist notable differences in drug metabolism that should be considered to prevent overprescribing.

Keywords: Gender; Psychotropic poisoning; Age; Length of stay; Discharges

Manuscript submitted October 8, 2020, accepted October 22, 2020

Published online December 9, 2020

a'Long School of Medicine, University of Texas Health San Antonio, TX, USA

${ }^{b}$ Department of Neurosurgery, University of Texas Health San Antonio, TX, USA

${ }^{\mathrm{c}}$ These authors contributed equally to this work.

${ }^{\mathrm{d} C o r r e s p o n d i n g ~ A u t h o r: ~ A l e e n a ~ V a r g a s, ~ L o n g ~ S c h o o l ~ o f ~ M e d i c i n e, ~ U n i v e r s i t y ~}$

of Texas Health San Antonio, TX 78229, USA.

Email: vargasac@livemail.uthscsa.edu

doi: https://doi.org/10.14740/jnr640

\section{Introduction}

Psychotropic agents are used to treat a variety of mental health conditions and include drug categories such as antidepressants, antipsychotics, anxiolytics, hypnotics, mood stabilizers and stimulants [1]. These agents are a growing cause of poisoning and mortality. Overdoses of psychotropic medication tend to be associated with higher ingested quantities when compared with other drugs of choice [2]. In particular, benzodiazepines have been identified as a leading cause in increasing hospitalizations [3].

Some patterns have been noted in the types of psychotropic agents commonly used in self-poisoning suicides. A Finnish study from 2017 found that the use of antidepressants in suicides doubled over their 23 -year study period. In addition, anxiolytics, hypnotics, sedatives and antipsychotics were found to be commonly used in suicides of patients with bipolar or unipolar depression. Inside the study period, antiepileptics were found to be used in one out of five total suicides between 2006 and 2010 [4].

Within the USA, ethnicity has been found to be a risk factor in the rate of deaths from psychotropic agents. The highest rate of mortality from psychotropic drugs occurs in non-Hispanic Native Americans, followed by non-Hispanic whites and Hispanics [5]. Given the risks associated with psychotropic agents and poisoning and the presence of differential risk in populations, there is a need to analyze trends in psychotropic agent poisoning in the male and female populations. Poisoning with psychotropic agents may be more likely in females due to factors such as provider prescribing preferences or higher rates of illnesses such anxiety, insomnia and chronic pain [6]. Our study looks at the trend associations between gender and psychotropic agent poisoning.

\section{Materials and Methods}

We performed a retrospective cohort study based on data from patients who experienced psychotropic agent poisoning from the years 1997 to 2014 gathered by the Healthcare Cost and Utilization Project (HCUP) from the Agency for Healthcare Research and Quality [7]. We analyzed several trends for poisoning by psychotropic agents using the CCS diagnosis code 241. Trends in the number of discharges, mean age, mean length of stay, mean hospital charges and number and percentage of in-hospital deaths were evaluated across the 18 years of the study, and each of these trends was then stratified by gender. The relative significance of the overall trends was evaluat- 


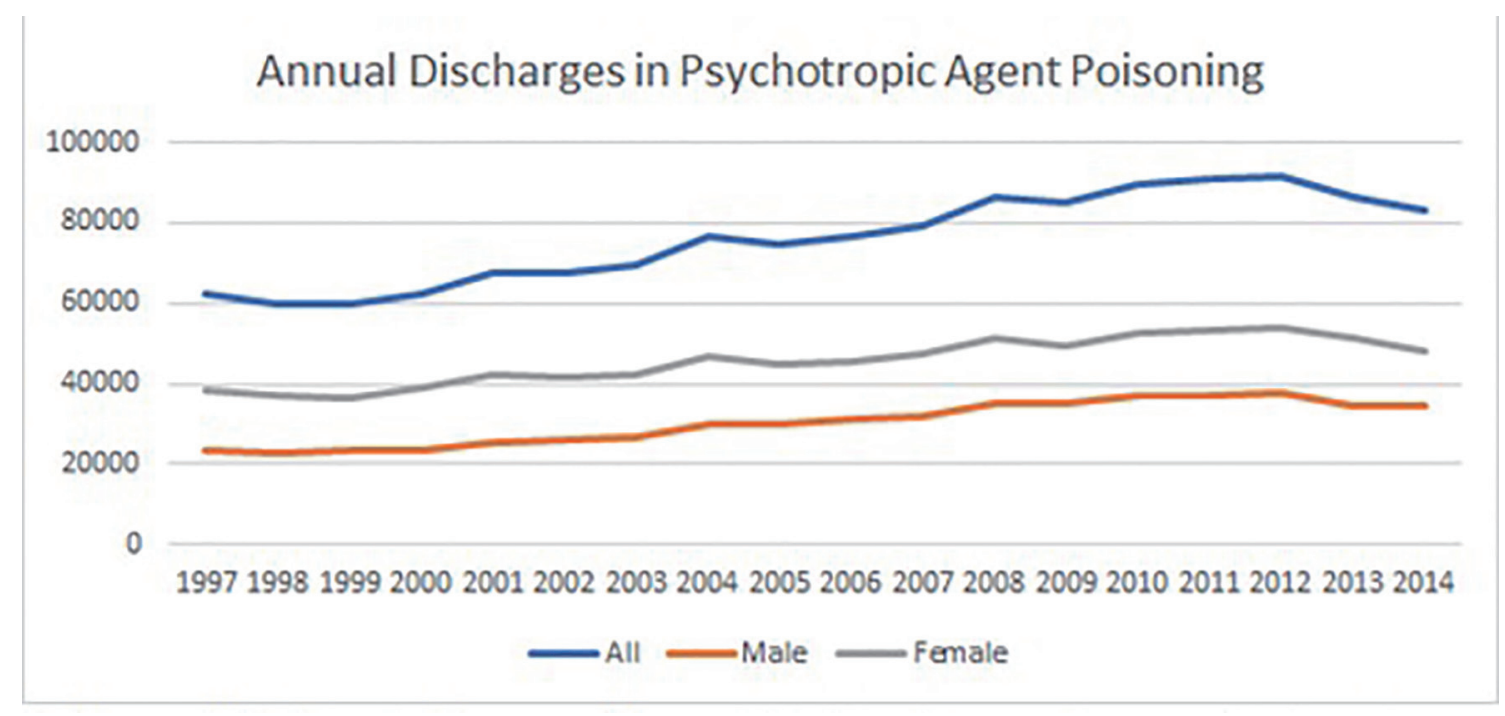

Figure 1. Trend of annual hospital discharges in psychotropic agent poisoning in the USA from 1997 to 2014.

ed by using $\mathrm{Z}$-test statistics and $\mathrm{P}$ values with a significance of less than 0.05 . The relative significance of the trends for male and female patients was also evaluated in the same manner. Finally, discharges and length of stay were compared between male and female patients for each year of the study using the same statistics. The University of Texas Health Science Center at San Antonio exempted our study from review by the Institutional Review Board.

\section{Results}

\section{Discharges}

Between 1997 and 2014, HCUP recorded a total of 1,368,649 psychotropic agent poisoning discharges. The overall number of discharges increased from 62,148 to 82,905 ( $\mathrm{P}<$ $0.001)$. Across the study period, the number of male discharges increased from 23,741 to $34,485(\mathrm{P}<0.001)$, and the number of female discharges increased from 38,407 to $48,380$ ( $\mathrm{P}<0.001)$. For every year of the study, female discharges were significantly greater than male discharges $(\mathrm{P}<$ 0.001, Fig. 1).

\section{Age}

Across the study period, average age at discharge increased from 37.36 to 40.85 years $(\mathrm{P}<0.001)$. Male age increased from 36.61 to $39.44(\mathrm{P}<0.001)$, while female age increased from 37.83 to $41.85(\mathrm{P}<0.001)$. For every year of the study,

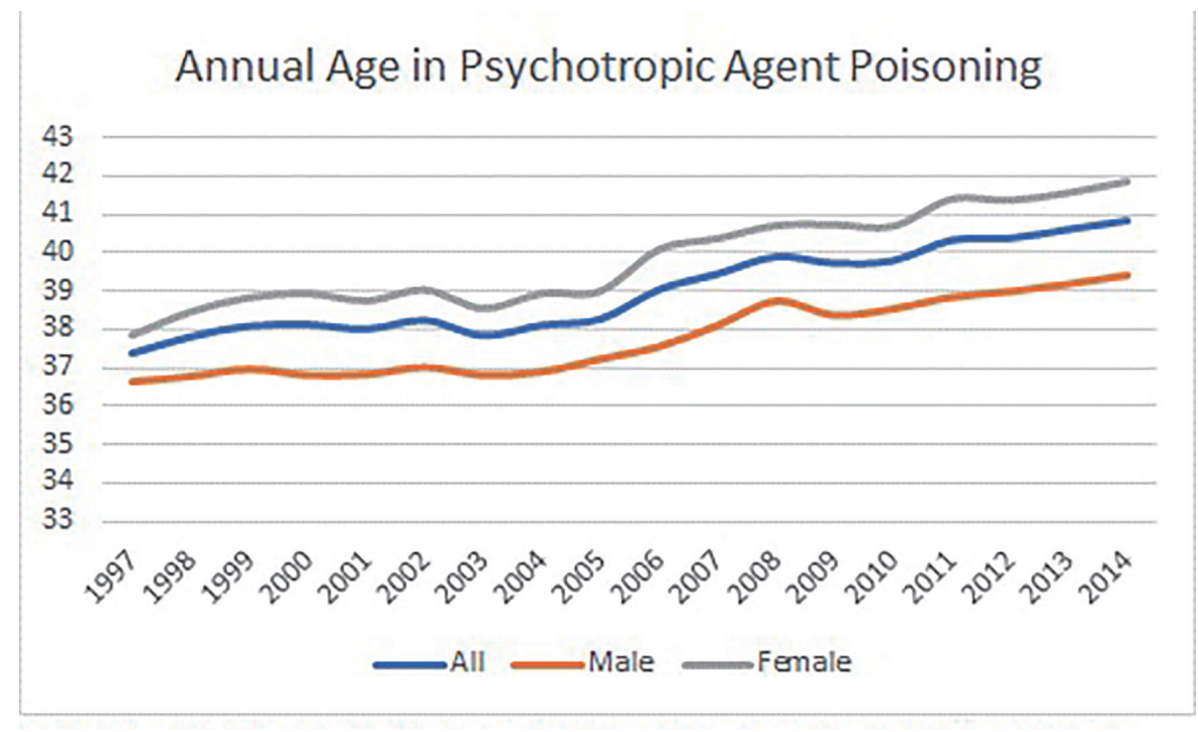

Figure 2. Trend of annual age in psychotropic agent poisoning in the USA from 1997 to 2014. 


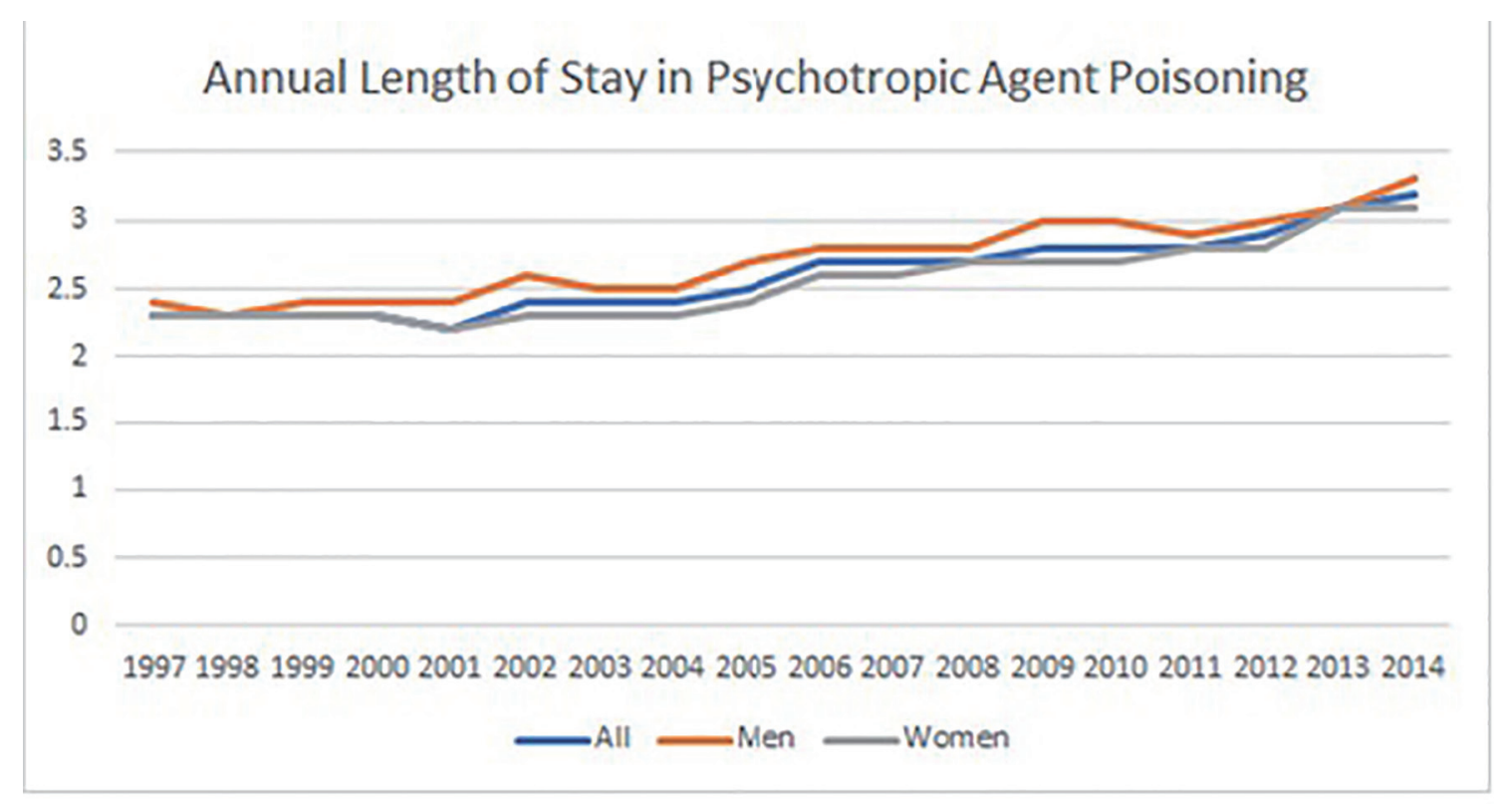

Figure 3. Trend of annual length of stay in psychotropic agent poisoning in the USA from 1997 to 2014.

age of female patients was significantly greater than age of male patients $(\mathrm{P}<0.001$, Fig. 2).

\section{Length of stay}

Across the study period, average length of stay increased from 2.3 to 3.2 days $(\mathrm{P}<0.001)$. The length of stay for male patients increased from 2.4 to 3.3 days $(\mathrm{P}<0.001)$, while the length of stay for female patients increased from 2.3 to 3.1 days $(\mathrm{P}<$
0.001, Fig. 3).

\section{Hospital charges}

Across the study period, hospital charges increased from $\$ 6,357$ to $\$ 27,892$ ( $\mathrm{P}<0.001)$. Charges for male patients increased from $\$ 7,011$ to $\$ 29,969(\mathrm{P}<0.001)$, while charges for female patients increased from $\$ 5,953$ to $\$ 26,420$ ( $\mathrm{P}<0.001$, Fig. 4).

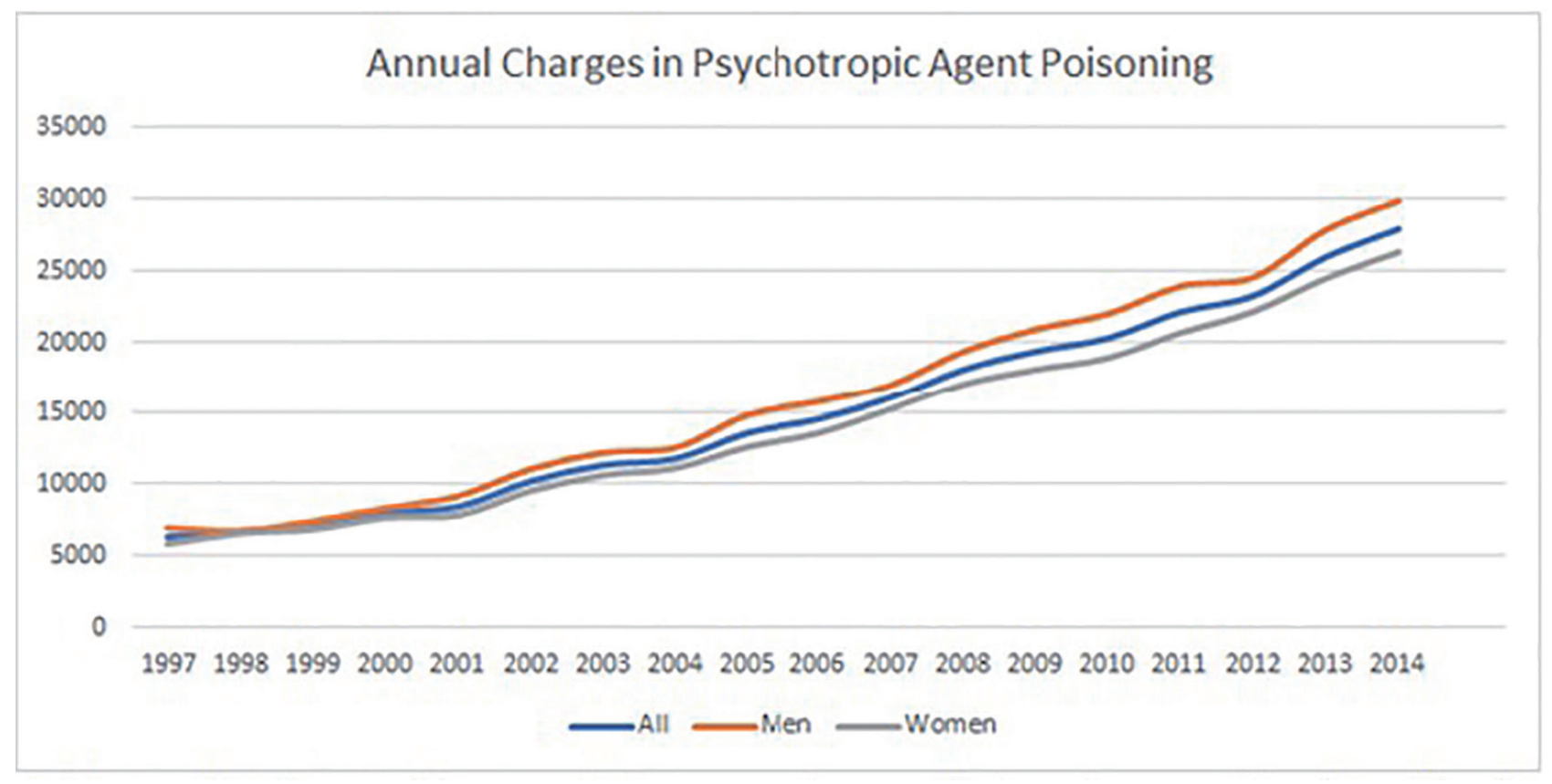

Figure 4. Trend of annual charges in psychotropic agent poisoning in the USA from 1997 to 2014. 


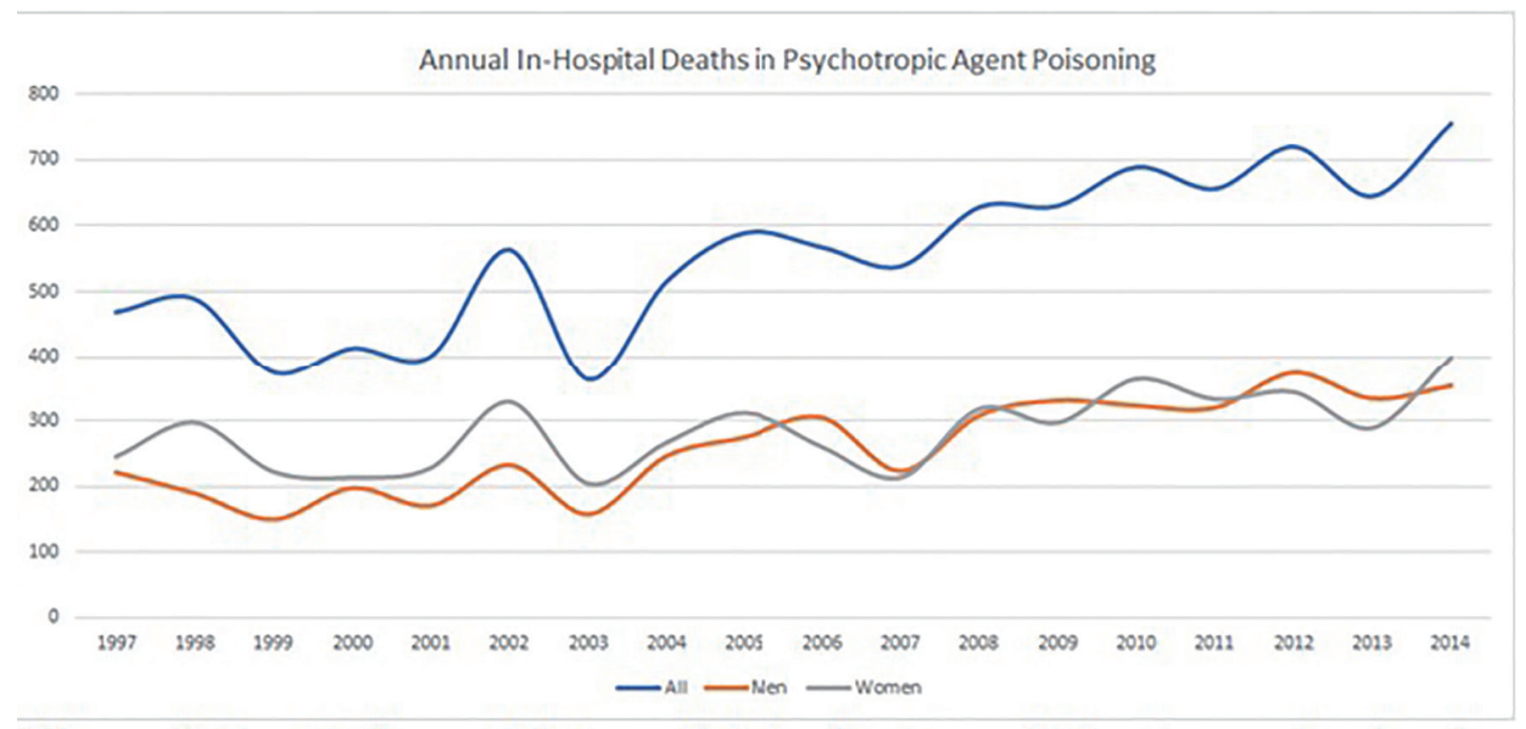

Figure 5. Trend of annual in-hospital deaths in psychotropic agent poisoning in the USA from 1997 to 2014.

\section{In-hospital deaths}

Across the study period, the number of in-hospital deaths increased from 468 to $755(\mathrm{P}<0.001)$. Deaths in male patients increased from 222 to $355(\mathrm{P}<0.05)$, while deaths in female patients increased from 246 to 400 ( $\mathrm{P}<0.01$, Fig. 5). No statistically significant increase was found for percentage of inhospital deaths (Fig. 6).

\section{Discussion}

A notable finding among the trends analyzed is the consistent and statistically significant difference between male and female annual discharges across the entire 18-year period. This larger number of discharges may suggest that psychotropic poisoning more commonly occurs in women than in men. A possible reason that women may experience psychotropic poisoning in higher numbers could be that a larger magnitude of women than men are being prescribed psychotropic medications. A study done in Ontario showed that female gender was associated with a greater likelihood of use of an outpatient mental health provider (adjusted odds ratio (OR): 1.7; 95\% confidence interval (CI): 1.2 - 2.4), a likelihood which was primarily attributed to mood/anxiety disorders [8]. These findings would be consistent with the overall higher prevalence of depression and anxiety disorders in women, with up

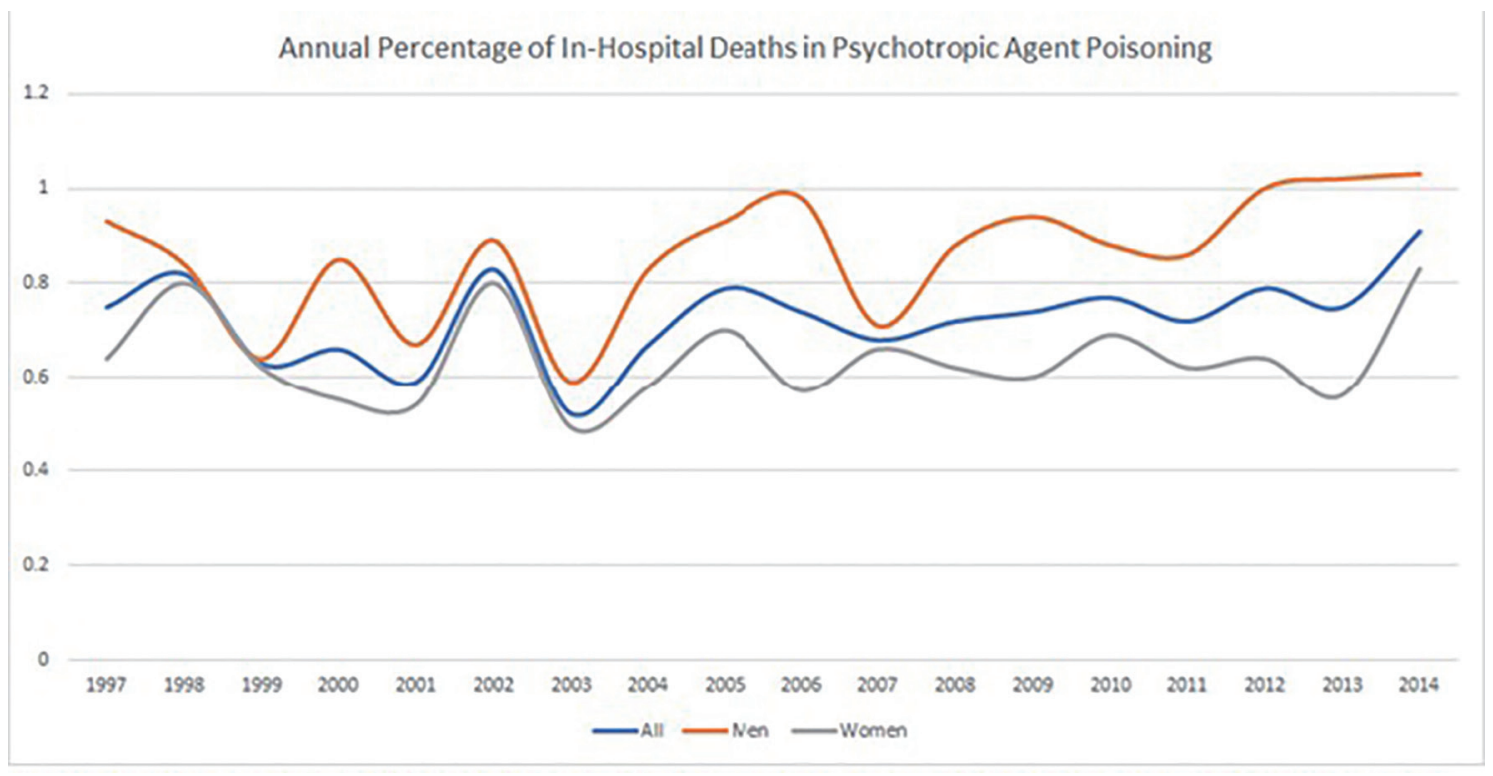

Figure 6. Trend of annual percentage of in-hospital deaths in psychotropic agent poisoning in the USA from 1997 to 2014. 
to twice the lifetime rate [9]. Additionally, when considering patients who have made office visits, women may be $55 \%$ more likely to be prescribed a psychotropic medication than men, with such medication primarily being antidepressants or anxiolytics [10].

It may be additionally worth considering the potential for gender differences in intentional self-poisoning in patients receiving psychotropic medications. It has been suggested that women may generally be at a higher risk of attempting suicide, and that the most common means is via pharmacologic drug abuse $[11,12]$. Ultimately, although women may be more likely to self-poison with psychotropic medication (OR: 1.2), they may be less susceptible when correcting for prescription rates (OR: 0.69) [13]. Nevertheless, this finding ultimately remains consistent with overall higher magnitude of hospital discharges related to psychotropic poisoning in women.

Pharmacologic dosing of psychotropics requires differential prescribing between men and women due to biological differences. One difference is in enzymatic activity, which causes psychotropic drugs to be metabolized at different rates. For example, the liver enzyme CYP1A2 is less active in women, leading to increased plasma concentrations of olanzapine and clozapine. Enzymes CYP1A2, 2C19, 2D6 and 3A4 have also shown differences in activity according to sex. In addition, women have a slightly decreased renal glomerular filtration rate and decreased activity of P-glycoprotein which could possibly lead to lower renal clearance of prescribed psychotropic medication. An example of this reduced clearance may be evident as a proportionally smaller decrease in glomerular filtration rate (GFR) $(10 \%)$ can result in a considerable increase in amisulpride plasma levels (40-50\%) in women [14]. Poor understanding of differing pharmacokinetics may leave women more susceptible to overprescribing.

Contributing to differential enzymatic activity is the increased likelihood for women to take other medications that interact with psychotropics. Antidepressants, mood stabilizers, analgesics, contraceptives and hormone replacements have been shown to compete with antipsychotics, particularly for the CYP2D6 enzyme. All of these classes of medication are taken more frequently by women than by men.

Biological differences in drug distribution throughout tissues also contribute to drug availability. Women tend to have less blood volume and higher body fat percentages than men. As lipophilic drugs, antipsychotic medications build up in fat tissue. As a result, women require longer maintenance dosing intervals due to already established reserves [15].

\section{Conclusions}

While analyzing several trends including annual discharges, hospital charges, mortality and age as they relate to psychotropic poisoning in the USA, a notable difference between male and female annual discharges is observed, which suggests a greater rate of psychotropic poisoning in female patients. There are multiple possible etiologies for this. The larger numbers may likely be partially attributed to higher psychotropic prescription rates among female patients and consequently greater exposure to the offending agent. However, other factors must also be considered, such as differing rates of suicidal intent and differing pharmacokinetics, in order to ensure proper prescribing and monitoring over the use of these medications.

\section{Acknowledgments}

None to declare.

\section{Financial Disclosure}

None to declare.

\section{Conflict of Interest}

None to declare.

\section{Informed Consent}

None applicable.

\section{Author Contributions}

Data collection and analysis were performed by Aleena Vargas and George Ormseth. Introduction, methods and results were written by Aleena Vargas, whereas discussion was written by George Ormseth. Corresponding sections in the abstract were written by each author. Dr. Ali Seifi assisted with graph design as well as with project guidance and supervision.

\section{Data Availability}

Any inquiries regarding supporting data availability of this study should be directed to the corresponding author.

\section{References}

1. Zohar J, Stahl S, Moller HJ, Blier P, Kupfer D, Yamawaki $\mathrm{S}$, Uchida $\mathrm{H}$, et al. A review of the current nomenclature for psychotropic agents and an introduction to the Neuroscience-based Nomenclature. Eur Neuropsychopharmacol. 2015;25(12):2318-2325.

2. Armstrong TM, Davies MS, Kitching G, Waring WS. Comparative drug dose and drug combinations in patients that present to hospital due to self-poisoning. Basic Clin Pharmacol Toxicol. 2012;111(5):356-360.

3. Coben JH, Davis SM, Furbee PM, Sikora RD, Tillotson RD, Bossarte RM. Hospitalizations for poisoning by prescription opioids, sedatives, and tranquilizers. Am J Prev Med. 2010;38(5):517-524.

4. Lofman S, Hakko H, Mainio A, Riipinen P. Affective dis- 
orders and completed suicide by self-poisoning, trend of using antidepressants as a method of self-poisoning. Psychiatry Res. 2017;255:360-366.

5. Goldstein E. Rise in mortality involving poisoning by medicaments other than narcotics, including poisoning by psychotropic drugs in different age/racial groups in the US. PLoS One. 2019;14(7):e0219711.

6. Bruno T, Pharr JR. Retrospective case series analysis of characteristics and trends in unintentional pharmaceutical drug poisoning by methadone, opioid analgesics, antidepressants and benzodiazepines in Clark County, NV 2009-13. J Public Health (Oxf). 2017;39(2):304-311.

7. https://www.hcup-us.ahrq.gov/

8. Rhodes AE, Goering PN, To T, Williams JI. Gender and outpatient mental health service use. Soc Sci Med. 2002;54(1):1-10.

9. Altemus M, Sarvaiya N, Neill Epperson C. Sex differences in anxiety and depression clinical perspectives. Front Neuroendocrinol. 2014;35(3):320-330.
10. Simoni-Wastila L. Gender and psychotropic drug use. Med Care. 1998;36(1):88-94.

11. Callanan VJ, Davis MS. Gender differences in suicide methods. Soc Psychiatry Psychiatr Epidemiol. 2012;47(6):857-869.

12. Tsirigotis K, Gruszczynski W, Tsirigotis M. Gender differentiation in methods of suicide attempts. Med Sci Monit. 2011;17(8):PH65-70.

13. Buckley NA, Dawson AH, Whyte IM, Hazell P, Meza A, Britt H. An analysis of age and gender influences on the relative risk for suicide and psychotropic drug self-poisoning. Acta Psychiatr Scand. 1996;93(3):168-171.

14. Haack S, Seeringer A, Thurmann PA, Becker T, Kirchheiner J. Sex-specific differences in side effects of psychotropic drugs: genes or gender? Pharmacogenomics. 2009;10(9):1511-1526.

15. Seeman MV. Gender differences in the prescribing of antipsychotic drugs. Am J Psychiatry. 2004;161(8):13241333. 